\title{
Comparisons of CTH Simulations with Measured Wave Profiles for Simple Flyer Plate Experiments
}

\author{
S. A. Thomas ${ }^{1}$ L. R. Veeser ${ }^{1,2} \cdot$ W. D. Turley ${ }^{3}$ R. S. Hixson ${ }^{1,2}$
}

Received: 25 February 2016/Accepted: 24 May 2016/Published online: 13 June 2016

(C) Society for Experimental Mechanics, Inc (outside the US) 2016

\begin{abstract}
We have conducted detailed 2-dimensional hydrodynamics calculations to assess the quality of simulations commonly used to design and analyze simple shock compression experiments. Such simple shock experiments also contain data where dynamic properties of materials are integrated together. We wished to assess how well the chosen computer hydrodynamic code could do at capturing both the simple parts of the experiments and the integral parts. We began with very simple shock experiments, in which we examined the effects of the equation of state and the compressional and tensile strength models. We increased complexity to include spallation in copper and iron and a solid-solid phase transformation in iron to assess the quality of the damage and phase transformation simulations. For experiments with a window, the response of both the sample and the window are integrated together, providing a good test of the material models. While CTH physics models are not perfect and do not reproduce all experimental details well, we find the models are useful; the simulations are adequate for understanding much of the dynamic process and for planning experiments. However, higher complexity in the simulations, such as adding in spall, led to greater differences between simulation and experiment. This comparison of simulation to experiment
\end{abstract}

S. A. Thomas

thomassa@nv.doe.gov

1 New Mexico Operations, National Security Technologies, LLC, Los Alamos, NM 87544, USA

2 Los Alamos National Laboratory, P. O. Box 1663, Los Alamos, NM 87545, USA

3 Special Technologies Laboratory, National Security Technologies, LLC, Santa Barbara, CA 93111, USA may help guide future development of hydrodynamics codes so that they better capture the underlying physics.

Keywords Hydrocode $\cdot$ Shock $\cdot$ Copper $\cdot$ Iron · CTH

\section{Introduction}

As computer power and speed have increased over the recent decades, hydrodynamic simulations of shock measurements have improved and have become more useful in comprehending experimental data and predicting the results of complex (integral) experiments. When detailed comparisons are made between the simulation of a complex experiment and the actual data, it is normal for there to be some differences, either minor or significant. Potential causes for difference include the numerical solution method used (Eulerian, Lagrangian, etc.), zone size chosen, artificial viscosity parameter, and so forth $[1,2]$.

There can also be issues with the physics models used in simulations, including possible pure phase uncertainties, incorrect phase boundary information, equilibrium/nonequilibrium effects, and so on. Typically strength models are constrained with low-strain-rate data and then applied to high-rate events, to which they may not actually apply. There may also be errors in the dynamic tensile (spall) description. The complex physics and metallurgical effects depend upon many parameters, only some of which are known. That makes spall difficult to model, and generally the models are overly simplistic. These issues have been described in detail elsewhere [3, 4].

In our experimental work to develop diagnostics for temperature, emissivity, phase change, and shock-induced damage experiments [5], we have made extensive use of the CTH hydrodynamic computer code from Sandia 
National Laboratories (SNL). CTH is a multi-dimensional Eulerian-Lagrangian hydrocode that has a large variety of material models and a useful, although limited, phase transformation capability [6].

We routinely use hydrodynamic simulations to guide experimental research where it can be difficult to do so using analytical methods. In this way we can design and plan both simple and more complex experiments before actually making measurements. Some experiments can be complicated to model and understand. We have found that the CTH code simulates some aspects of our experiments quite well, but for complicated problems, such as phase transformations or spall, the calculations do not always agree well with data. We have carefully examined the capability of CTH to model simpler, planar shock experiments to determine the code's limitations and see whether there are ways it (or its input parameters) can be improved. We proceeded to model velocimetry data from several planar gun shots on copper and iron using original velocimetry data from Los Alamos National Laboratory (LANL). Although these experiments were done to obtain very fundamental data, their data also contain more complex information. Some of this data has not been published previously, so we provide information here on experimental configuration, materials used, geometry, and dimensions.

CTH is readily available from SNL to US users; support provided to users is excellent. $\mathrm{CTH}$ is adaptable to numerous geometries and material properties, runs on several platforms, and is not difficult to learn to use. As a result, it is widely used in the shock physics community. Although this study is specific to CTH, it is our hope that by illustrating some of the complications of simulationcode operation, our work will be instructive to users of other hydrocodes as well.

By working with relatively simple experiments, we reduce the number of effects being integrated so that we are able to isolate problems more easily. For example, even though there are edge effects that must be considered, planar experiments present a simplified geometry and a condition of uniaxial strain. Likewise, single-material experiments eliminate problems that arise when convolving multiple material responses. Eventually we added the complexity of spall damage and an iron phase transformation to our study.

\section{Velocimetry Data}

In choosing data to simulate, we began with gun shots on copper because it has no phase changes in the regime of interest and is generally thought to be a metal with simple dynamic response. Copper is commonly used as a standard in impedance-match equation-of-state (EOS) experiments, and therefore it has been studied extensively both experimentally and theoretically. We expected that its models for yield strength, plasticity, spall, etc., are relatively well developed and so would provide us a good test of CTH's basic capabilities. Later we modeled iron, which has a phase transformation from $\alpha$ to $\varepsilon$ around $13 \mathrm{GPa}$ (a stress that can be easily reached with many guns) and a reversion around $10 \mathrm{GPa}$.

Table 1 includes shot parameters, all of which were fielded at LANL and some of which were published previously by LANL researchers. All of the velocimetry data were collected with VISAR systems (velocity interferometer system for any reflector) [7].

Experiments CuSPALL, Cu16, and 56-97-14 have not been previously described in detail, and so we give relevant information here. The copper material used in CuSPALL and $\mathrm{Cu} 16$ was OFHC (oxygen-free, high conductivity, c10100 specification) with $99.99 \%$ purity. This metal has about $0.0005 \%$ oxygen and a density of $8.93 \mathrm{~g} / \mathrm{cm}^{3}$. Samples were annealed at $600{ }^{\circ} \mathrm{C}$ for $1 \mathrm{~h}$, resulting in an average grain size of 40-80 $\mu \mathrm{m}$. For experiment 56-97-14 the metal used was HY100 steel with a nominal initial density of $7.87 \mathrm{~g} / \mathrm{cm}^{3}$. This steel is approximately $96 \%$ pure and is relatively well characterized for its mechanical properties. In addition, the data clearly show the presence of the $\alpha-\beta$ phase transition. The high-purity iron metal used in experiments 031999 b and 990311 has been well documented previously [8]. Chemical analysis and average grain size are given in that reference, and its measured density was $7.85 \mathrm{~g} / \mathrm{cm}^{3}$.

\section{Modeling Results}

\section{Copper Spall}

Data for experiment CuSPALL are shown in Fig. 1. The measured peak stress in the initial shock for the CuSPALL experiment is $5.73 \mathrm{GPa}$. The approximate spall strength for this experiment, ignoring corrections, is

$\sigma_{\text {spall }} \sim 1 / 2 \rho_{0} C_{b} \Delta u_{f s}$

For this experiment, this approximation yields a spall strength of about $1.37 \mathrm{GPa}$. Here $\rho_{O}$ is the copper density, $C_{b}$ is its Eulerian bulk sound speed $(3.93 \mathrm{~km} / \mathrm{s})$, and $\Delta u_{f s}$ is the free surface velocity decrease between the peak immediately after shock release and the spall minimum.

As is true for all shock experiments, we must get the EOS right to have any hope of understanding the more complex phenomena. In simulating this experiment, we used a simple Mie-Gruneisen EOS combined with the Johnson-Cook (J-C) viscoplastic model for the strength 
Table 1 Experiment parameters

\begin{tabular}{|c|c|c|c|c|c|c|c|}
\hline Sim.\# & $\begin{array}{l}\text { Name } \\
\text { (date) }\end{array}$ & $\begin{array}{l}\text { Peak stress } \\
(\mathrm{GPa})\end{array}$ & Experiment & Impactor type and thickness (mm) & $\begin{array}{l}\text { Target } \\
\text { type and thickness }(\mathrm{mm})\end{array}$ & Window & $\begin{array}{l}\text { Impactor } \\
\text { velocity } \\
(\mathrm{m} / \mathrm{s})\end{array}$ \\
\hline 1 & $\begin{array}{l}\text { CuSPALL } \\
(8 / 26 / 93)\end{array}$ & 5.7 & Copper spall & $\begin{array}{l}\text { Copper } \\
1.78\end{array}$ & $\begin{array}{l}\text { Copper } \\
4.00\end{array}$ & None & 308 \\
\hline 2 & $\begin{array}{l}\mathrm{Cu} 16 \\
(8 / 28 / 96)\end{array}$ & 11.5 & Copper release & Sapphire 2.811 & $\begin{array}{l}\text { Copper } \\
5.083\end{array}$ & Sapphire & 508 \\
\hline 3 & $56-97-14$ & 16.0 & $\begin{array}{l}\text { Iron } \\
\text { release }\end{array}$ & $\begin{array}{l}\text { Sapphire } \\
3.192\end{array}$ & $\begin{array}{l}\text { Iron } \\
1.081\end{array}$ & Sapphire & 762 \\
\hline 4 & $\begin{array}{l}\text { 031999b } \\
(3 / 19 / 99)\end{array}$ & 10.2 & $\begin{array}{l}\text { Iron spall [8] } \\
(\alpha \text { phase) }\end{array}$ & $\begin{array}{l}\text { Iron } \\
2.06\end{array}$ & $\begin{array}{l}\text { Iron } \\
4.06\end{array}$ & None & 590 \\
\hline 5 & $\begin{array}{l}990311 \\
(3 / 11 / 99)\end{array}$ & 16.5 & $\begin{array}{l}\text { Iron spall }[8] \\
\text { ( } \varepsilon \text { phase })\end{array}$ & $\begin{array}{l}\text { Sapphire } \\
3.19\end{array}$ & $\begin{array}{l}\text { Iron } \\
2.20\end{array}$ & None & 810 \\
\hline
\end{tabular}

Experimental data for simulations 4 and 5 are from [8]

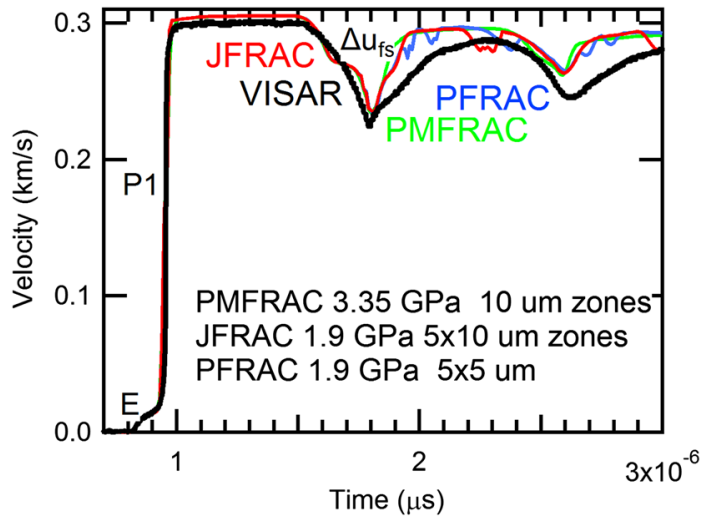

Fig. 1 Copper symmetric shock and spall at the free surface. The curves are a comparison between the free surface velocity measurement and $\mathrm{CTH}$ calculations for experiment CuSPALL

[9]. How well CTH calculates the EOS is shown in the peak free surface velocity in the flat top of this wave profile (Fig. 1) between 1.0 and $1.5 \mu \mathrm{s}$. In this case there is excellent agreement between experiment and simulation, indicating that CTH accurately calculates both the initial compression and the complete release that occurs at the free surface when the initial shock reflects there. The precursor wave at around $0.8 \mu$ s is the small elastic wave that precedes the main (bulk) shock wave. The CTH simulation also models this well, and excellent agreement is observed. The parameters for the $\mathrm{J}-\mathrm{C}$ viscoplastic model are apparently well chosen to represent the elastic shock response of copper.

Most of the issues in modeling this experiment have to do with the spall response. We employed three spall models. In CTH notation, PFRAC is the nominal threshold spall model, in which spall occurs as soon as the tension reaches the value defined by the PFRAC parameter. This model is sometimes called $\mathrm{P}_{\min }$ because it specifies a minimum value of pressure (tension) beyond which the code assumes spall occurs. The PMFRAC model spreads the damage over several time steps to reduce extraneous ringing in the velocity. For a ductile material like copper, PMFRAC is intended to account for the strain that can accumulate before void coalescence and separation of the material at the spall surface. A third spall model, the $\mathrm{J}-\mathrm{C}$ damage model (not the same as the $\mathrm{J}-\mathrm{C}$ viscoplastic model) is called JFRAC. Damage is calculated by integrating the strain rate. For copper the three models give comparable results for the free surface velocity up to the spall minimum, although the spall strength values required by $\mathrm{CTH}$ to reproduce the data are larger than the 1.2-1.4 GPa that is typically measured for supported (flat-topped) shocks of comparable strength [10-12]. PFRAC and JFRAC need spall strength parameters of about $1.9 \mathrm{GPa}$, and PMFRAC requires $3.35 \mathrm{GPa}$ for this problem. We chose not to try to use the TulerButcher fracture model to simulate the copper data.

Note that the deviation of all of the calculational results from the data immediately following the first release ( $\mathrm{t} \sim 1.6 \mu \mathrm{s})$ is due to the simulation calculating a more distinct elastic/plastic release than occurs in the data. The 'smeared-out' elastic/plastic release observed in the data is commonly referred to as quasi-elastic release. This effect has been looked at previously [13], and it is clear that CTH does not capture this release response.

The deviation of calculated results from the data at time $>1.7-1.8 \mu$ s is caused by reflections from the free surface of the elastic wave trapped in the spall scab; i.e. ringing in the spall scab. In the experiment these reflections damp out quickly, but the simulation retains its memory. In this 
ringing in the spall scab, the three models differ slightly, and all of them calculate a velocity recovery from the spall minimum that is faster than measured. We have observed a zone size effect in this region, especially for PFRAC and JFRAC. As we decrease the zone sizes, the simulations for these two models approach that for PMFRAC. The best results used a small axial zone size, $10 \mu \mathrm{m}$, to capture the shock rise, the spall feature, and the ringing. Making the zones even smaller, $<5 \mu \mathrm{m}$, however, seems to cause another problem; the spall minimum becomes too shallow, requiring an even larger spall strength parameter. We point this out for completeness, even though we do not attempt to discuss numerical convergence issues. This is only intended to note that zone sizes will affect some details in the calculated results and should be considered.

\section{Copper Release (Cu16)}

Figure 2 shows results for experiment $\mathrm{Cu} 16$, in which a sapphire flyer impacts a copper target, and the copper shock wave moves into a z-cut sapphire window. A flat-top shock begins to release gradually at $1.9 \mu$ s when the rarefaction fan from the back of the impactor begins to overtake the shock. In the simulation, CTH must convolve the EOS of $\mathrm{Cu}$ together with that of sapphire to obtain the proper interface particle velocity, $u_{i}$. It does this well, and we see only a small disagreement in the elastic wave and the peak particle velocity state. Note, however, that the sapphire EOS parameters provided in CTH are not meant for single-crystal, z-cut sapphire, and those parameters give wrong velocities in this problem. We used instead the EOS parameters of Barker and Hollenbach [14], $U_{s}=$ $11.19+1.0 \mathrm{u}_{\mathrm{p}}$, where $U_{s}$ is the shock speed and $\mathrm{u}_{\mathrm{p}}$ the particle speed. A Gruneisen gamma value of 1.5 was used as well. $\mathrm{CTH}$ is able to calculate the interface state in

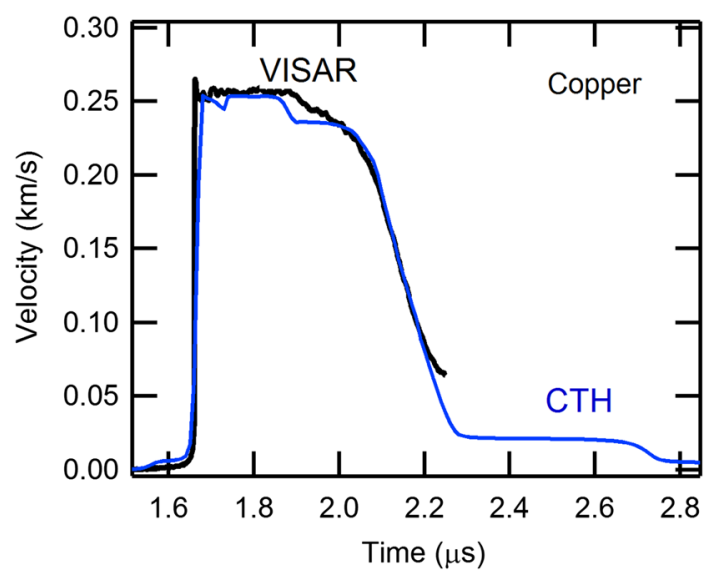

Fig. 2 Copper symmetric shock and release into sapphire. The curves are a comparison between the free surface velocity measurement and $\mathrm{CTH}$ calculations for experiment $\mathrm{Cu} 16$ release very well. With the exception of the release-side strength signature $(1.9-1.95 \mu \mathrm{s}), \mathrm{CTH}$ follows the release side of this wave profile down to relatively low particle velocity, in good agreement with the experiment. The disagreement in the release-side strength signature, both here and in the spall experiment ("Copper Spall" section), is not surprising, as the $\mathrm{J}-\mathrm{C}$ strength model apparently does not include the physics of quasi-elasticity. Note that this experiment is done below the elastic limit for z-sapphire, so the flyer and window responses in this experiment are purely elastic.

\section{Iron Release (56-97-14)}

Figure 3 shows the VISAR data and three CTH simulations for an iron release experiment at sufficient stress to induce the alpha-epsilon $(\alpha-\varepsilon)$ solid-solid phase change on initial compression. The sapphire window has a shock impedance that is close to that of iron, so there is almost no reflection at the interface. The geometry was designed with a thick window and thin sample to produce a phase reversion shock in the iron before the shock could leave the window (and damage its free surface). For this experiment we follow Barker [15] notation, where $\mathrm{E}$ is the elastic wave, P1 is the first plastic wave, $\mathrm{P} 2$ is the transformed plastic or phase transition wave, ER is the arrival of the first elastic relief wave, and EPR is the arrival of the partial release of the elastic wave from the back of the impactor. The interface velocity shows the shock beginning to release at $2.15 \mu \mathrm{s}$. When the reversion to $\alpha$ phase occurs, it causes a release shock $(2.3 \mu \mathrm{s})$ because the $\alpha$-phase material has a larger specific volume than $\varepsilon$.

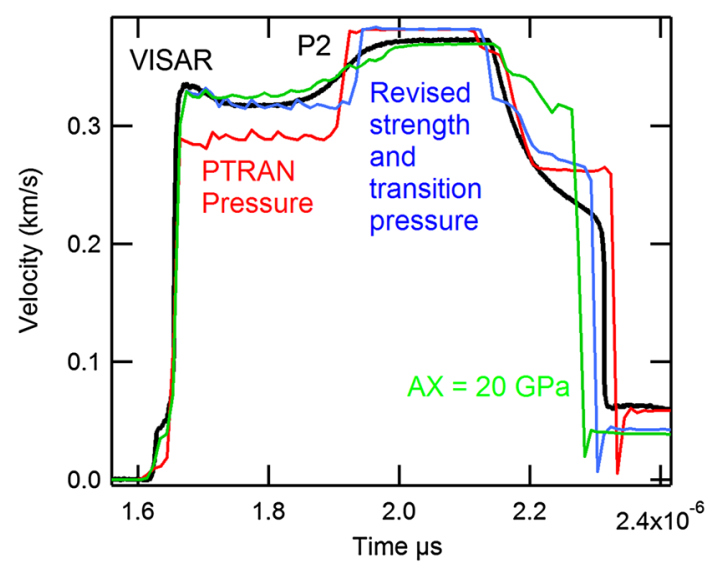

Fig. 3 Results for a shock in iron that releases into a z-cut sapphire window. Black VISAR data; red simulation using CTH with PTRAN phase-transformation model; blue iron strength and $\varepsilon$ phase parameters changed as outlined in the text; green same $\varepsilon$ parameters with transformation rate slowed in proportion to the fraction of untransformed material remaining 
The phase change was simulated using the PTRAN EOS option for iron in CTH (red curve, Fig. 3). We used CTH library values for the Gruneisen EOS parameters for both the $\alpha$ and the $\varepsilon$ phases. The initial calculation (red curve) shows the elastic precursor $(1.6 \mu \mathrm{s})$, the P1 plastic wave $(1.65-1.95 \mu \mathrm{s})$, the P2 wave $(1.95-2.15 \mu \mathrm{s})$, the start of release $(2.15 \mu \mathrm{s})$, and the phase-reversion shock $(2.3 \mu \mathrm{s})$. The elastic precursor has about the right velocity, but its strength, which can depend greatly on the impurities in the sample, does not agree with the measurements when using a strength model for pure iron. However, this experiment was actually done on HY100 low-alloy steel, which has a higher yield strength than pure iron. Part of this disagreement may also be because low strain rate (Kolsky-bar pressures or lower) data are used to construct the $\mathrm{J}-\mathrm{C}$ strength model, and it may not be capturing the real dynamics at the much higher strain rates in this experiment. In other words, we suspect that no shock compression data were used to constrain this $\mathrm{J}-\mathrm{C}$ model. For iron this is probably a relatively minor issue, but for materials with a higher elastic limit, it becomes more serious. To improve the elastic precursor, we changed from the $\mathrm{J}-\mathrm{C}$ strength model for iron to that of steel by calling the values from the CTH library, but we kept the PTRAN EOS model for iron. Using the steel strength model brings the precursor amplitudes into better agreement (green and blue curves).

The plastic shock measurements show a small velocity decrease beginning at $1.7 \mu \mathrm{s}$, after the P1 wave breaks out into the window and a very different and more gradual approach to full stress from P1 $(1.9 \mu \mathrm{s})$ than the initial calculation (red curve). The initial calculation also shows a much lower phase transition stress (red curve) than the data. The simulation shows a phase transition stress that is in agreement with the Bancroft [16] and Barker [15] results, but the data imply a phase transition stress higher than the original measurements. Barker noted that the observed transition stress decreases with sample thickness for samples up to a few millimeters thick, so the difference is caused by the thin $(1 \mathrm{~mm})$ sample used. Therefore, we increased the transition pressure to $13.8 \mathrm{GPa}$, the approximate pressure for a $1 \mathrm{~mm}$ sample and $17.0 \mathrm{GPa}$ driving stress; see Fig. 10 of Ref. [9]. The calculation with 13.8 $\mathrm{GPa}$ transition pressure and the strength change to steel are shown as the blue line. They are an improvement and agree well with the experimental results of Barker [15].

Published results using the Sandia WONDY code to simulate iron gun shots show that the phase transformation slows as the transformation proceeds and leaves less $\alpha$ phase material available for the transformation [8]. To slow the phase transformation in CTH we used the parameter AX, the derivative of the phase transformation pressure with respect to the $\varepsilon$-phase fraction, which defaults to 0 . Empirically we found that a value of $\mathrm{AX}=20 \mathrm{GPa}$ is roughly right for this experiment. Including that value along with the increased phase change stress and higher yield strength gives the green curve (Fig. 3).

The calculations show the release shock correctly and at roughly the correct time, indicating that the phase change is reversible and that $\mathrm{CTH}$ is simulating the shock and sound speeds more or less accurately. However, the calculated phase reversion happens at nearly the same pressure as the $\alpha-\varepsilon$ phase change, not at the lower pressure characteristic of the experimental data. The PTRAN model does not have the physics in it to be able to capture the hysteresis in the phase change and its reversion.

\section{Iron Free Surface Below the Phase Transition}

Figure 4 shows velocimetry from a spall experiment 031999b (black) and three CTH calculations. The measured spall strength is $\sim 1.9 \mathrm{GPa}$. Two of the calculations are for LANL's SESAME EOS (green), also given in the CTH data parameters, and the EOS from the Marsh [17] compendium (red), both with PFRAC spall strengths of $1.9 \mathrm{GPa}$. The third (blue) uses the SESAME EOS and a smaller spall strength, $1.5 \mathrm{GPa}$. The calculations all fail to match the data, even in the flat-top region, $0.8-1.3 \mu \mathrm{s}$, where the calculated velocity is several percent too low. Again, as described in "Iron Release(56-97-14)" section we used the steel viscoplastic model, and again it shows roughly the right yield strength but not the elastic wave decay pullback before the P1 wave $(0.7 \mu \mathrm{s})$. The calculations with the SESAME EOS show the tensile process beginning too early, and the Marsh EOS gives the wrong spall signature, even using the smaller spall strength.

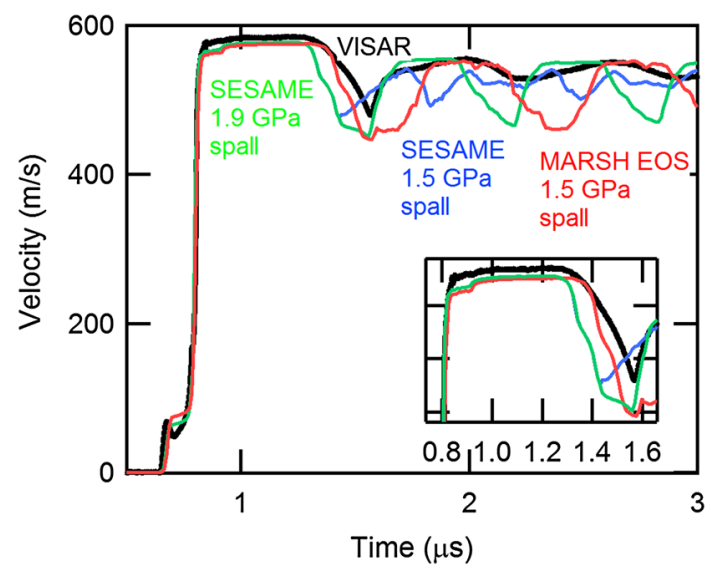

Fig. 4 Iron symmetric shock below the $\alpha-\varepsilon$ phase transformation and release into air. None of the simulations reproduce the pullback in the elastic precursor $(0.7 \mu \mathrm{s})$, the velocity of the flat-top shock $(0.8-1.3 \mu \mathrm{s})$, or the spall parameters 


\section{Iron Spall from the $\varepsilon$ Phase}

CTH calculations are compared to velocimetry for an iron spall shot (990311) that occurs above the $\alpha-\varepsilon$ phase change in Fig. 5. Here we used the revised PTRAN parameters for strength, pressure, and transition speed, as described in "Iron Release (56-97-14)" section for iron release, and we tried the same three fracture models as for copper (see the three colored curves). Including the AX parameter to slow the calculated phase transition again helps to get the P1-P2 transition right, but it causes an erroneous delay in the beginning of the release (see the small bump centered at $1 \mu \mathrm{s}$ ).

JFRAC is clearly not appropriate for iron, which is less ductile than copper. PMFRAC also gives a spall strength that is too small. PFRAC gives the best agreement with the measured spall strength of about 2.6 GPa and also shows the proper damping after fracture. PFRAC is remarkably good, especially in light of the difficulty in calculating the spall below the phase change. Note that the reflection that occurs at the iron target free surface causes a release back to zero stress. Similarly, the release wave coming back from the rear surface on the impactor is a release to very low stress. Because the phase transition in iron is known to be reversible and to happen quickly through a rarefaction shock not present here, the spall almost certainly is happening from the alpha phase. If we compare the pullback amplitudes between experiments above and below the transition, we see general agreement. We see what may be the beginnings of a 'PIR' wave, as described in detail by Barker [15].

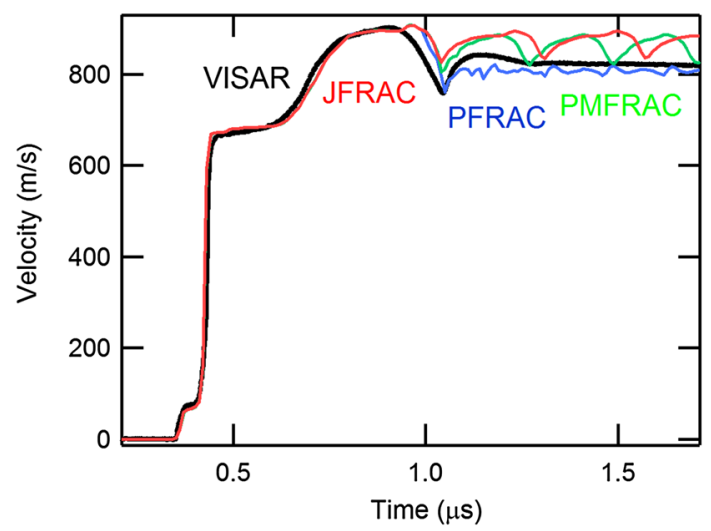

Fig. 5 Measurements and calculations for iron that spall in the $\varepsilon$ phase. As described in the text, PFRAC (blue curve) gives the best agreement with the measurements. All the calculations used $2.7 \mathrm{GPa}$ spall strength

\section{Discussion}

Although many of the models in CTH are not perfect and do not reproduce all of the experimental details well, the code can produce simulations adequate for many of the details in our flyer plate experiments. Agreement with copper measurements is generally good where the EOS plays a significant role, but is less so for the dynamic damage process. Considerable work has been done to understand spall behavior [18], but the spall process is very complex and depends upon both details of the dynamic loading process and material properties.

For the copper window experiment, we see very good agreement when the best model parameters for z-sapphire are used. The main difference observed between experiment and simulation is due to the dislocation governed yielding process and reversal when stress is removed. This physics is not in the models used. CTH did a good job capturing the EOS governed details, and it was able to successfully integrate together the dynamic responses of copper and sapphire.

For iron, as expected, agreement between data and simulation is not as good. The presence of the $\alpha-\varepsilon$ phase change complicates overall dynamic response. A variation of parameters was needed to capture details of the phase transition, both for the stress amplitude and the rate of transition. The variation of phase transition stress with sample thickness is a complication that the PTRAN model is not able to capture. But the PTRAN model does have a parameter that allows variation of transition rate that allowed us to bring the data and simulation into better agreement. This kind of fundamental data allows that parameter to be set. In addition, the Mie-Gruneisen EOS for the $\alpha$ phase leads to noticeable difference between data and simulation. We also note that the location in both stress and time of the reverse transition varied as we varied model parameters, with only fair agreement with data. But, importantly, CTH did capture the rarefaction shock in the reversion process. Finally, as for copper, details of the spall process are not captured well.

Through this research, we have seen that zone size in the simulations is important, and numerical convergence studies should always be considered. We have also seen that the EOS used in CTH, particularly Mie-Gruneisen, can have uncertainties that lead to systematic differences between simulation and reality (experiment). We observed good agreement for copper but a few percent differences for iron. Additionally, strength models can represent the dynamic strength processes in dynamic events well, or they can fall short. This depends on the complexity of the fundamental material yielding process, the deformation rate, and the fidelity of the strength model used. We tried 
the Steinberg-Guinan strength model for copper with results that are very similar to J-C. For the J-C models, the authors themselves point out that they use no fundamental shock compression data to constrain the model, so extrapolating this model up in strain rate must be done with some caution. Finally, spall is the most difficult property to capture numerically. We have found that a simple $\mathrm{P}_{\min }$ model captures the depth of the spall pullback, and consequently the approximate spall strength for both copper and iron, reasonably well. However, the value required for $\mathrm{P}_{\min }$ is not always the same as the strength measured in fundamental plate impact experiments.

\section{Conclusion}

In this work we have begun a process of obtaining a better understanding of how well numerical hydrodynamic codes do in simulating geometrically simple flyer plate experiments. We chose experiments that were originally designed to yield information that can be used in a forward sense to constrain physics models for EOS, dynamic compressive strength, and dynamic tensile strength. We then compared these time-resolved data with simulations. These experiments contain data that are both very fundamental (Hugoniot elastic limit, peak particle velocity, time of first release, etc.) and more complex/integral (spall, phase transition, etc.). Some of the experiments also contain data that came from integrating the response of two materials, the target and the window, to test how well a hydrodynamic code can replicate this complexity.

Our results show that the CTH code, which is designed to handle shock compression problems, does well overall. For the parts of the wave profiles that contain fundamental information, such as peak particle velocity and time of first release, it does remarkably well. CTH also does very well at integrating together the responses of copper and sapphire in simulating the $\mathrm{Cu} 16$ experiment. For the more complex parts of the wave profiles, such as the details of the spall process and the phase transition in iron, comparisons are not as good. Not surprisingly, we also found that some numerical parameters, such as zone size, can be important as well.

From this work we improved our understanding of the strengths and weaknesses of this important code, and we are reporting them to share this information. Overall we believe there is a need for improved physics models, at least in the areas where we found discrepancies, and probably also in other areas, such as dynamic compressive strength.

Acknowledgments We gratefully acknowledge the help of John Vorthman in performing the experiments used here. We also acknowledge very useful and stimulating conversations with Gerald Stevens and Brandon La Lone. We furthermore acknowledge that these experiments were done at Los Alamos National Laboratory, with the support of the U.S. Department of Energy. This manuscript has been authored by National Security Technologies, LLC, under Contract No. DE-AC52-06NA25946 with the U.S. Department of Energy. The United States Government retains and the publisher, by accepting the article for publication, acknowledges that the United States Government retains a non-exclusive, paid-up, irrevocable, world-wide license to publish or reproduce the published forms of the manuscript, or allow others to do so, for United States Government purposes. The U.S. Department of Energy will provide public access to these results of federally sponsored research in accordance with the DOE Public Access Plan (http://energy.gov/downloads/doe-publicaccess-plan). DOE/NV/25946-2781.

\section{References}

1. VonNeumann J, Richtmyer RD (1950) A method for the numerical calculation of hydrodynamic shocks. J Appl Phys 21:232

2. Richtmyer RD, Morton KW (1967) Difference methods for initial-value problems, 2nd edn. Wiley, New York

3. Kerley GI (2009) Equation of state and constitutive models for numerical simulations of dust impacts on the solar probe. Kerley Technical Services: Report KTS09-1

4. Kerley GI (1993) Multiphase equation of state for iron. Sandia National Laboratory: Report SAND93-0027:UC-410

5. La Lone BM, Stevens GD, Turley WD, Holtkamp DB, Iverson AJ, Hixson RS, Veeser LR (2013) Release path temperatures of shock-compressed tin from dynamic reflectance and radiance measurement. J Appl Phys 114:063506

6. McGlaun JM, Thompson SL, Elrick MG (1990) CTH-A three dimensional shock wave physics code. Int J Impact Eng 10:351-360

7. Barker LM, Hollenbach RE (1972) Laser interferometer for measuring high velocities of any reflecting surface. J Appl Phys 43:4669-4675

8. Veeser LR, Gray GT III, Vorthman JE, Rodriguez PJ, Hixson RS, Hayes DB (2000) High pressure response of a high-purity iron. AIP Conf Proc 505:73-76

9. Johnson GR Cook WH 1983 A constitutive model and data for metals subjected to large strains, high strain rates and high temperatures. Proceedings of the Seventh International Symposium on BallisticsThe Hague 541548

10. Koller DD, Hixson RS, Gray GT, Rigg PA, Addessio LB, Cerreta EK, Maestas JD, Yablinsky CA (2005) Influence of shock-wave profile on dynamically induced damage in high-purity copper. J Appl Phys 98:103518

11. Hixson RS, Gray GT, Rigg PA, Addessio LB, Yablinsky CA (2004) Dynamic damage investigation using triangular waves. AIP Conf Proc 706:469-472

12. Escobedo JP, Dennis-Koller D, Cerreta EK, Patterson BM, Bronkhorst CA, Hansen BL, Tonks D, Lebensohn RA (2011) Effects of grain size and boundary structure on the dynamic tensile response of copper. J Appl Phys 110:033513

13. Johnson JN, Hixson RS, Gray GT III, Morris CE (1992) Quasielastic release in shock-compressed solids. J Appl Phys 72:429

14. Barker LM, Hollenbach RE (1970) Shock-wave studies of PMMA, fused silica, and sapphire. J Appl Phys 41:4208

15. Barker LM, Hollenbach RE (1974) Shock wave study of the $\alpha-\varepsilon$ phase transition in iron. J Appl Phys 45:4872

16. Bancroft D, Peterson EL, Minshall S (1956) Polymorphism of iron at high pressure. J Appl Phys 27:291-298

17. Marsh SP (1980) LASL shock Hugoniot data. University of California Press, Berkeley

18. Antoun T, Seaman L, Curran DR, Kanel GI, Razorenov SV, Utkin AV (2003) Spall fracture. Springer, New York 